\title{
Successful extracorporeal liver dialysis for the treatment of trimethoprim-sulfamethoxazole-induced fulminant hepatic failure
}

\author{
Choon Ta $\underline{\mathrm{Ng}}^{1}$, MBBS, MRCP, Chee Kiat $\underline{\mathrm{Ta}}^{1}$, FRCP, FAMS, Choon Chiat $\underline{\mathrm{Oh}}^{2}$, MBBS, MrCP,
} Jason Pik Eu Chang ${ }^{1}$, MRCP, FAMS

\begin{abstract}
Trimethoprim-sulfamethoxazole (TMP-SMZ) is a commonly used antibiotic that has been associated with drug rash with eosinophilia and systemic symptoms (DRESS) syndrome. DRESS syndrome is characterised by fever, rash, lymphadenopathy, eosinophilia and one or more major organ involvement. Although rare, TMP-SMZ is a recognised cause of fulminant hepatic failure. We report a 17-year-old Chinese male adolescent who presented with fever, myalgia, generalised maculopapular rash and lymphadenopathy after taking TMP-SMZ for acne vulgaris. He subsequently developed hepatic encephalopathy and was worked up for urgent liver transplantation. He responded well to extracorporeal liver dialysis (originally intended as a bridging therapy) and subsequently recovered without the need for liver transplantation. This case report highlights the importance of early recognition of TMP-SMZ-induced DRESS syndrome and the need for early discontinuation of the drug in the affected patient. Extracorporeal liver dialysis and transplantation should be considered in the management of TMP-SMZ-induced fulminant hepatic failure.
\end{abstract}

Keywords: drug rash with eosinophilia and systemic symptoms (DRESS), fulminant hepatic failure, molecular adsorbents recirculation system liver dialysis, trimethoprim-sulfamethoxazole

\section{INTRODUCTION}

Trimethoprim-sulfamethoxazole (TMP-SMZ) is a commonly used antibiotic in clinical practice for the treatment of communityacquired, methicillin-resistant Staphylococcus aureus skin and soft tissue infections. Besides that, TMP-SMZ also has an important role in the prophylaxis and treatment of Pneumocystis jiroveci pneumonia in immunocompromised patients. ${ }^{(1)}$ TMP-SMZ-induced drug rash with eosinophilia and systemic symptoms (DRESS) syndrome is a rare but recognised complication, occurring in $0.01 \%-0.1 \%$ of patients, ${ }^{(2)}$ and can cause fulminant hepatic failure. We describe a case of TMP-SMZinduced DRESS syndrome associated with fulminant hepatic failure in an adolescent who was successfully managed with extracorporeal liver dialysis.

\section{CASE REPORT}

A 17-year-old Chinese male adolescent presented to our emergency department with fever, myalgia and a nonpruritic maculopapular rash over his trunk and neck. He had developed nonpruritic, confluent, erythematous macules and papules after taking TMP-SMZ (80 mg TMP, $400 \mathrm{mg}$ SMZ) for 28 days. The macules and papules first appeared over the neck and chest, and progressively spread to his limbs and the rest of his trunk within the next few days. He had been prescribed TMP-SMZ and isotretinoin by a general practitioner for nodular cystic acne vulgaris. Despite symptomatic treatment by the general practitioner, the patient's rash and fever did not subside, thus prompting a visit to our emergency department.

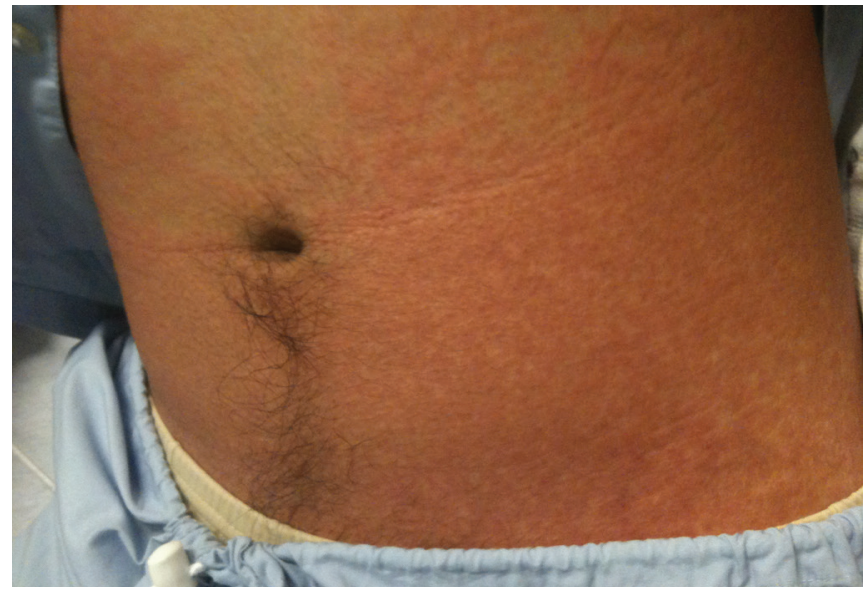

Fig. 1 Photograph shows generalised, confluent, erythematous maculopapular rash over the trunk.

The patient had no significant medical history and no glucose-6-phosphate dehydrogenase deficiency. Other than TMP-SMZ and isotretinoin, he occasionally took paracetamol for fever. He was not on any recreational medication or herbal supplement, and denied any alcohol intake. He had recently travelled to Batam (an island in the Riau Archipelago) two months ago and engaged in water sports. However, he did not recall having any insect bite and denied any sexual intercourse. Clinical examination upon admission revealed him to be alert but jaundiced, with a fever of $38.4^{\circ} \mathrm{C}$ and a confluent, erythematous, maculopapular rash over his trunk, face, arms and legs (Fig. 1). His mucosal surfaces (including the eyes,

${ }^{1}$ Department of Gastroenterology and Hepatology, ${ }^{2}$ Department of Dermatology, Singapore General Hospital, Singapore

Correspondence: Dr Jason Chang Pik Eu, Consultant, Department of Gastroenterology and Hepatology, Singapore General Hospital, Outram Road, Singapore 169608. jason.chang@sgh.com.sg 
Table I. Laboratory results of the patient.

\begin{tabular}{|c|c|c|c|c|c|c|}
\hline Parameter & Normal range & At admission & Before MARS & After MARS & On discharge & $\begin{array}{l}\text { Outpatient } \\
\text { follow-up }\end{array}$ \\
\hline Total protein (g/L) & $62-82$ & 65 & 44 & 54 & 64 & 68 \\
\hline Albumin (g/L) & $37-51$ & 38 & 21 & 22 & 28 & 39 \\
\hline Bilirubin $(\mu \mathrm{mol} / \mathrm{L})$ & $3-24$ & 51 & 287 & 293 & 136 & 24 \\
\hline Alkaline phosphatase (U/L) & $32-103$ & 223 & 179 & 150 & 172 & 68 \\
\hline $\operatorname{ALT}(U / L)$ & $7-36$ & 1,660 & 1,859 & 564 & 105 & 27 \\
\hline AST (U/L) & $15-33$ & 1,249 & 701 & 254 & 94 & 30 \\
\hline PT (s) & $<11.2$ & 12.2 & 26.0 & 32.5 & 11.9 & 11.5 \\
\hline
\end{tabular}

ALT: alanine transaminase; AST: aspartate transaminase; MARS: molecular adsorbent recirculating system; PT: prothrombin time

mouth and genitalia) were unaffected and there was no evidence of skin detachment or positive Nikolsky's sign. He had bilateral cervical and inguinal lymphadenopathy, but abdominal examination was unremarkable and there was no hepatic flap.

Laboratory tests showed a white blood cell count of $8.4 \times$ $10^{9} / \mathrm{L}$ (normal range $4.0-10.0 \times 10^{9} / \mathrm{L}$ ), raised absolute eosinophil count of $0.67 \times 10^{9} / \mathrm{L}$ (normal range $0.04-0.44 \times$ $10^{9} / \mathrm{L}$ ), elevated serum bilirubin at $51 \mu \mathrm{mol} / \mathrm{L}$ (normal range 3-24 $\mu \mathrm{mol} / \mathrm{L}$ ), alanine transaminase (ALT) at 1,660 U/L, aspartate transaminase (AST) at 1,249 $\mathrm{U} / \mathrm{L}$ and a prothrombin time (PT) of $12.2 \mathrm{~s}$ (Table I). Notably, his serum acetaminophen level was not elevated. Serological studies for hepatitis A, B and C viruses were nonreactive. Tests for human immunodeficiency virus, human herpesvirus 6 (HHV6), Epstein-Barr virus, cytomegalovirus, herpes simplex virus, dengue virus and autoantibodies (i.e. antinuclear antibody, antimitochondrial antibody, smooth muscle antibody, antineutrophil cytoplasmic antibody, antiliver/kidney microsome antibody type 1) were negative. Anti-hepatitis E virus (HEV) immunoglobulin (Ig) M was present, but both anti-HEV IgG and HEV ribonucleic acid (RNA) were nonreactive. Serum and urine toxicology tests were unremarkable. Wilson's disease was also excluded. However, ultrasonography of the abdomen showed mild, nonspecific fatty liver changes, which could be a radiological manifestation of severe hepatitis.

The initial impression was that of a drug-induced liver injury, both TMP-SMZ and isotretinoin were immediately discontinued upon admission. The patient was started on intravenous vitamin $\mathrm{K}$ daily for eight days and acetylcysteine infusion for five days. As the patient was pyrexic, he was empirically covered with intravenous ceftriaxone and acyclovir after consultation with the infectious disease team. In view of the patient's persistent fever, ceftriaxone was escalated to piperacillin-tazobactam after two days. He was also administered lactulose and intravenous fresh frozen plasma transfusion. His skin biopsy showed features consistent with drug exanthem (interface dermatitis, spongiosis, scattered apoptotic cells and inflammatory cells consisting of lymphocytes and eosinophils) (Fig. 2). In view of the patient's cervical and inguinal lymphadenopathies, urgent consultation with the haematologist was sought. However, the results of both peripheral blood film and flow cytometry excluded any underlying lymphoproliferative disorder.

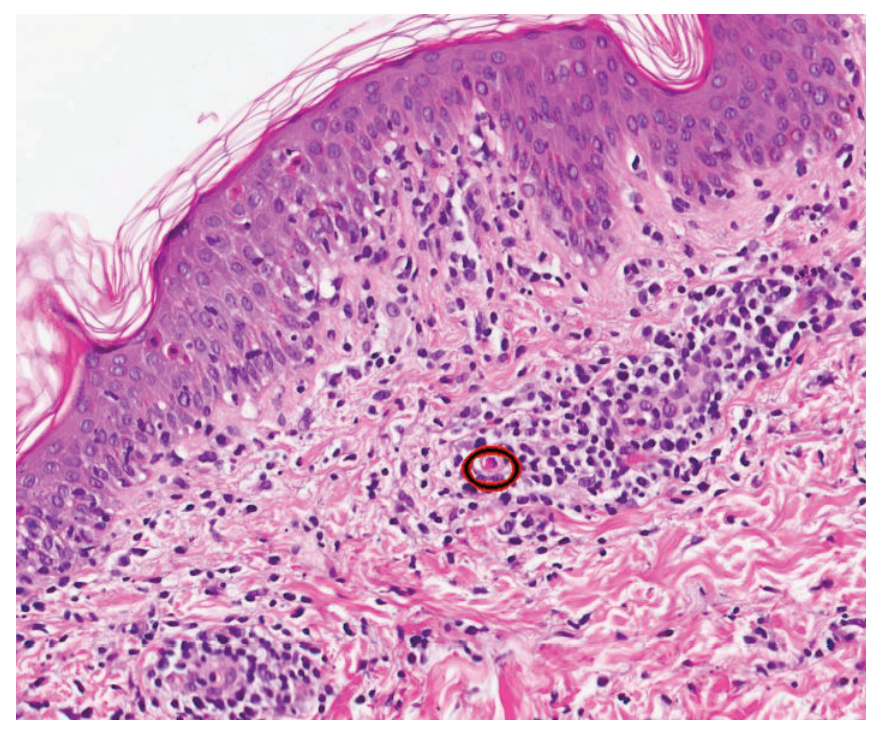

Fig. 2 Photomicrograph of skin punch biopsy shows interface dermatitis, spongiosis, scattered apoptotic cells, inflammatory cells, including lymphocytes and eosinophils, and an eosinophil (circle) within the dermal inflammatory infiltrate (Haematoxylin \& eosin, $\times 60$ ).

Despite discontinuation of TMP-SMZ, elevation of transaminases and bilirubinaemia continued (total serum bilirubin $327 \mu \mathrm{mol} / \mathrm{L}$, AST 2,054 U/L, ALT 2,862 U/L, PT $39.9 \mathrm{~s}$ ), and the patient became increasingly disoriented on Day 8 of hospitalisation. His maculopapular exanthem also worsened and almost covered his entire body surface area, but there was no involvement of the mucosal surfaces and no evidence of skin detachment. Clinically, the patient was in grade III hepatic encephalopathy, and a decision was made for him to undergo molecular adsorbent recirculating system (MARS) extracorporeal liver dialysis. As he fulfilled the King's College Criteria ${ }^{(3)}$ for consideration of liver transplantation, workup for both living and deceased donor liver transplant was initiated. He was not administered systemic steroids due to a lack of evidence of its efficacy in drug reactions such as this and a possible risk of aggravating a latent virus infection. ${ }^{(2)}$ He was intubated for airway protection and underwent two cycles of MARS liver dialysis in the intensive care unit over a course of two days. His condition improved, and he became more alert and oriented. Both clinical and psychoanalytical (number connection) tests revealed no residual hepatic encephalopathy. His rash subsequently resolved, with remaining brown postinflammatory hyperpigmentation and desquamation (Fig. 3). The patient was removed from the 
Table II. Summary of patients with TMP-SMZ-induced fulminant hepatic failure reported in the literature.

\begin{tabular}{|c|c|c|c|c|c|c|}
\hline Source, Year & Age (yrs) & Gender & Clinical symptoms & Treatment & Outcome & Additional drug exposure \\
\hline Bell et al, 2010 (1) & 9 & Male & $\begin{array}{l}\text { Fever, headache, } \\
\text { neck pain }\end{array}$ & $\begin{array}{l}\text { Withdrawal of } \\
\text { medication }\end{array}$ & Survived & Acetaminophen \\
\hline Ilario et al, 2000(4) & 60 & Female & Fever & $\begin{array}{l}\text { Withdrawal of } \\
\text { medication }\end{array}$ & Died & Phenytoin \\
\hline Alberti-Flor et al, 1989(5) & 26 & Male & $\begin{array}{l}\text { Maculopapular rash } \\
\text { over trunk, epigastric } \\
\text { abdominal pain }\end{array}$ & $\begin{array}{l}\text { Withdrawal of } \\
\text { medication }\end{array}$ & Died & Nitrofurantoin, doxycycline \\
\hline Zaman et al, $2003^{(7)}$ & 23 & Male & $\begin{array}{l}\text { Myalgia, fever, rash } \\
\text { over neck and chest }\end{array}$ & $\begin{array}{l}\text { Liver } \\
\text { transplantation }\end{array}$ & Survived & Nil \\
\hline Horak et al, 1984(13) & Not known & Not known & Not known & $\begin{array}{l}\text { Resin } \\
\text { haemoperfusion }\end{array}$ & Survived & Not known \\
\hline Ransohoff and Jacobs, $1981^{(14)}$ & 70 & Male & $\begin{array}{l}\text { Jaundice, rash over } \\
\text { trunk and leg }\end{array}$ & Neomycin & Died & Acetaminophen \\
\hline
\end{tabular}

liver transplant waiting list and discharged well on Day 25 of hospitalisation. His liver function tests returned to normal two months later and the rash completely resolved.

\section{DISCUSSION}

Fulminant hepatic failure associated with TMP-SMZ is a rare but recognised adverse drug reaction, occurring as soon as one day, or up to six weeks, after first exposure. ${ }^{(1,2)}$ TMP-SMZ can induce cholestatic or mixed hepatocellular-cholestatic injury, and in some instances, hepatic necrosis and granulomatous change. ${ }^{(4)}$ Reactive metabolites of SMZ, rather than the parent drug, are believed to be responsible for the idiosyncratic drug reaction. ${ }^{(5,6)}$ There may be a genetic predisposition in patients who are slow acetylators, possibly due to impairment in normal elimination of toxic metabolites. ${ }^{(7)}$ In $0.01 \%-0.1 \%$ of patients, TMP-SMZ induces DRESS syndrome with a significant mortality rate of up to $10 \%$. $^{(2,6)}$ DRESS syndrome is characterised by fever, rash, lymphadenopathy, eosinophilia and involvement of one or more organs, ${ }^{(2,6,8)}$ and typically occurs one to five weeks after initiation of the offending drug. ${ }^{(5)}$ Our patient had confluent erythematous maculopapular rash, fever and elevated transaminases four weeks after taking TMP-SMZ. The results of our employment of the Naranjo criteria, ${ }^{(9)}$ which predicts the likelihood of a medication causing an allergic reaction, indicated that TMP-SMZ was the most likely cause of the adverse drug reaction in our patient.

Markedly raised transaminases are usually found in patients with TMP-SMZ-induced fulminant hepatic failure, with previous case reports detailing ALT and AST levels ranging from 2,000 U/L to $19,520 \mathrm{U} / \mathrm{L}$ and $957 \mathrm{U} / \mathrm{L}$ to $9,400 \mathrm{U} / \mathrm{L}$, respectively. ${ }^{(4,5)}$ Morimotoetalalsoreported thatTMP-SMZ-inducedhypersensitivity syndrome may be associated with reactivation of HHV6. ${ }^{(2)}$ In our patient, polymerase chain reaction (PCR) for HHV6 DNA was negative. Recently, HEV testing has also been recommended in patients with drug-induced liver injury ${ }^{(9)}$ Although serologic testing for anti-HEV IgM with an enzyme-linked immunosorbent assaybased test (Genelabs Diagnostics, Singapore) was positive in our patient, HEV RNA by reverse transcriptase PCR and anti-HEV IgG were nonreactive. Follow-up serological tests for anti-HEV IgM and

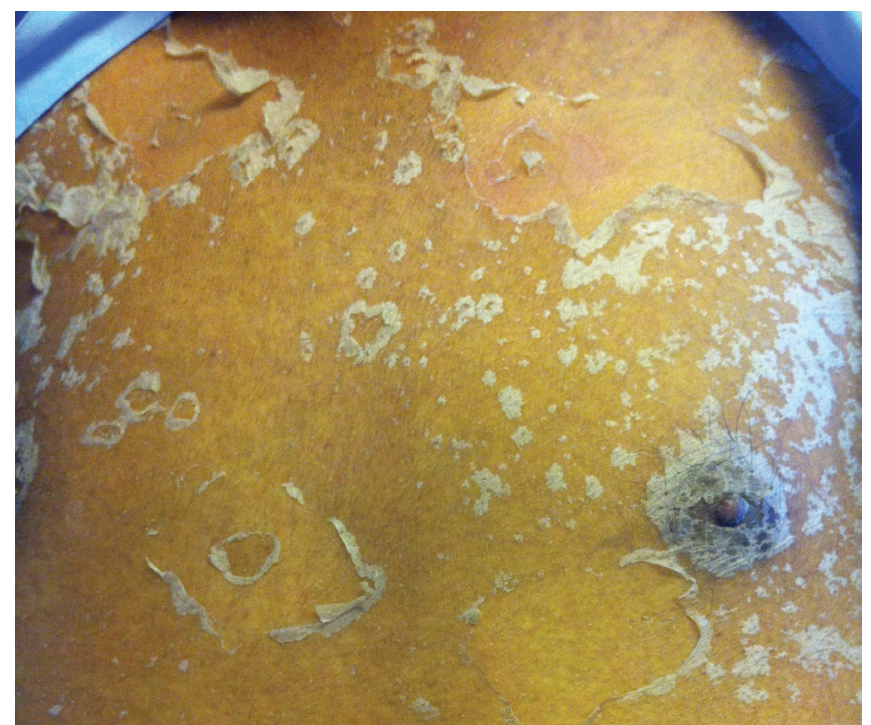

Fig. 3 Photograph shows postinflammatory hyperpigmentation with desquamation over the trunk.

anti-HEV IgG at two weeks and one month revealed positivity for anti-HEV IgM but remained nonreactive for anti-HEV IgG. Rheumatoid factor IgM, which can cause a false-positive anti-HEV IgM, was also nonreactive. Our patient's clinical presentation and time course of exposure suggested that he may have had a false-positive anti-HEV IgM result, instead of a bona fide acute HEV infection confounding his drug-induced liver failure. False-positive anti-HEV IgM has been reported to occur in $1.9 \%$ of patients. ${ }^{(10)}$ Our patient was administered acetaminophen for fever, but he denied any overdosage and his blood acetaminophen level was within the therapeutic range. He had also taken isotretinoin for acne five days prior to admission. While isotretinoin can result in elevation of transaminases, it is not usually associated with drug hypersensitivity syndrome. ${ }^{(11)}$ To our knowledge, isotretinoininduced fulminant liver failure has not been reported. We did not perform transjugular liver biopsy in our patient, as the clinical picture was consistent with a drug-induced liver injury and the procedural risks in the setting of the patient's grossly deranged coagulation profile outweighed the benefits. Considering the temporal sequence of events and the fact that all virus markers 
were negative, TMP-SMZ was the most likely cause of fulminant hepatic failure in our patient. Although most patients with TMP-SMZ-induced fulminant hepatic failure improve with discontinuation of the drug, some may require resin haemoperfusion or liver transplantation. ${ }^{(1,5,7,8,12)}$ Deaths from TMP-SMZ-induced fulminant hepatic failure have also been reported. ${ }^{(4,5)}$ Table II summarises the reported cases of patients with TMP-SMZ-induced fulminant hepatic failure. ${ }^{(1,4,5,7,7,13,14)}$

In patients with suspected TMP-SMZ-induced hepatotoxicity, the offending drug should be discontinued immediately. Patients suffering from fulminant hepatic failure with progressive encephalopathy and worsening coagulopathy should be on the priority list for liver transplantation. ${ }^{(7)}$ In the interim, patients may benefit from extracorporeal liver dialysis. The use of MARS in fulminant hepatic failure is generally indicated when there is significant hepatic encephalopathy (West Haven grade 2) in the setting of raised bilirubin and international normalised ratio of more than 1.5. Although the use of MARS in fulminant hepatic failure is associated with a significant reduction in total bilirubin levels and marked improvement in hepatic encephalopathy, it has no significant impact on all-cause mortality and acts mainly as a bridging therapy to liver transplantation. ${ }^{(13,15)}$ Our patient was placed on the priority list for liver transplantation in view of his worsening encephalopathy, and MARS liver dialysis was initiated as a bridging therapy to liver transplantation. As he made excellent recovery after two cycles of MARS, he was subsequently removed from the liver transplant list. Thus, MARS could have been a bridge to the naturally occurring recovery process of the liver in our patient.

In conclusion, physicians should recognise that TMP-SMZ is known to cause DRESS syndrome and can be associated with fulminant hepatic failure. In such instances, the offending medication should be discontinued immediately. Patients should be closely monitored for signs of cutaneous progression to Stevens-Johnson syndrome, toxic epidermal necrolysis and deterioration in liver function. Patients who develop hepatic failure should be referred to a tertiary hepatology and transplant service. Lifesaving extracorporeal liver dialysis and liver transplantation may be necessary and should be initiated early.

\section{REFERENCES}

1. Bell TL, Foster JN, Townsend ML. Trimethoprim-sulfamethoxazole-induced hepatotoxicity in a pediatric patient. Pharmacotherapy 2010; 30:539.

2. Morimoto T, Sato T, Matsuoka A et al. Trimethoprim-sulfamethoxazoleinduced hypersensitivity syndrome associated with reactivation of human herpesvirus-6. Intern Med 2006; 45:101-5.

3. O'Grady JG, Alexander GJ, Hayllar KM, Williams R. Early indicators of prognosis in fulminant hepatic failure. Gastroenterology 1989; 97:439-45.

4. Ilario MJ, Ruiz JE, Axiotis CA. Acute fulminant hepatic failure in a woman treated with phenytoin and trimethoprim-sulfamethoxazole. Arch Pathol Lab Med 2000; 124:1800-3.

5. Alberti-Flor JJ, Hernandez ME, Ferrer JP, Howell S, Jeffers L. Fulminant liver failure and pancreatitis associated with the use of sulfamethoxazoletrimethoprim. Am J Gastroenterol 1989; 84:1577-9.

6. Knowles SR, Uetrecht], Shear NH. Idiosyncratic drug reactions: the reactive metabolite syndromes. Lancet 2000; 356:1587-91.

7. Zaman F, Ye G, Abreo KD, Latif S, Zibari GB. Successful orthotopic liver transplantation after trimethoprim-sulfamethoxazole associated fulminant liver failure. Clin Transplant 2003; 17:461-4.

8. Kouklakis G, Mpoumponaris A, Zezos P, et al. Cholestatic hepatitis with severe systemic reactions induced by trimethoprim-sulfamethoxazole. Ann Hepatol 2007; 6:63-5.

9. Naranjo CA, Busto $U$, Sellers EM, et al. A method for estimating the probability of adverse drug reactions. Clin Pharmacol Ther 1981; 30:239-45.

10. Davern TJ, Chalasani N, Fontana RJ, et al. Acute Hepatitis E Infection Accounts for Some Cases of Suspected Drug-Induced Liver Injury. Gastroenterology 2011; 141:1665-72.

11. Lin CC, Wu JC, Chang TT, et al. Diagnostic value of immunoglobulin G (IgG) and IgM anti-hepatitis E virus (HEV) tests based on HEV RNA in an area where hepatitis E is not endemic. J Clin Microbiol 2000; 38:3915-8.

12. Zane LT, Leyden WA, Marqueling AL, Manos MM. A population-based analysis of laboratory abnormalities during isotretinoin therapy for acne vulgaris. Arch Dermatol 2006; 142:1016-22.

13. Horak J, Mertl L, Hrabal P. Severe liver injuries due to sulfamethoxazoletrimethoprim and sulfamethoxydiazine. Hepatogastroenterology 1984; 31:199-200.

14. Ransohoff DF, Jacobs G. Terminal hepatic failure following a small dose of sulfamethoxazole-trimethoprim. Gastroenterology 1981; 80:816-9.

15. Vaid A, Chweich H, Balk EM, Jaber BL. Molecular adsorbent recirculating system as artificial support therapy for liver failure: a meta-analysis. ASAIO J 2012; 58:51-9. 